\title{
Erratum a: I segni di Graefe e Stellwag nell'ipertiroidismo
}

\author{
Roberto Toni
}

Pubblicato online: 23 ottobre 2014

(C) Springer International Publishing AG 2014

\section{Erratum a: l'Endocrinologo (2014) 15: 186-189 DOI 10.1007/s40619-014-0052-7}

Il testo a pagina 188 , riga 12 : “... del nervo oculomotore (via afferente del riflesso)" deve essere modificato in "... del nervo trigemino (via afferente del riflesso)".
Il testo nella legenda di Figura 2, pagina 188 deve essere modificato come segue:

1. “...(prima edizione tedesca, 1867); (C) frammenti dal paragrafo..." deve essere sostituito con: ... (prima edizione tedesca, 1867) con frammenti dal paragrafo.

2. “...facies ipertiroidea; (D) frequenza del battito palpebrale..." deve essere sostituito con: “... facies ipertiroidea; (C)"
La versione online dell' articolo originale può essere trovata al doi:10.1007/s40619-014-0052-7.

R. Toni $(\varangle)$

Unità di Antropometria e Medicina delle Costituzioni, Centro

Interdipartimentale di Medicina dello Sport e dell'Esercizio

Fisico, Università degli Studi di Parma, Parma, Italia

e-mail: roberto.toni@unipr.it

R. Toni

Accademia delle Scienze dell'Istituto di Bologna, Bologna, Italia

e-mail: roberto.toni@unibo.it

R. Toni

Department of Medicine, Division of Endocrinology, Diabetes and Metabolism, Tufts Medical Center-Tufts University School of Medicine, Boston, MA, USA

e-mail: roberto.toni@tufts.edu 